\title{
Enfisema sub-cutáneo en procedimientos de cirugía oral. Revisión de la literatura
}

\author{
Subcutaneous emphysema in oral surgery procedure. Review of literature \\ Elías Alejandro Cabanillas Taco ${ }^{1, a, b, c,}$, Víctor Manuel Arrascue Dulanto 2,d,e,f.
}

\section{RESUMEN}

Objetivo: Determinar la causa más frecuente del enfisema sub-cutáneo en Cirugía oral, así como los factores de riesgo, diagnostico y tratamiento. Material y métodos: Búsqueda exhaustiva de la literatura a través de PubMed \& Cochrane del 2009 al 2018. Se utilizaron los reportes sobre procedimientos quirúrgicos. Se excluyeron los casos ocasionados por trauma y tratamientos de conducto. Se encontró 21 reportes que cumplían con nuestros criterios de inclusión. Resultados: Se encontró que 14 de los 21 casos estaban relacionados con el uso de pieza de mano de alta velocidad. Otros casos incluyeron: 4 por el uso de la Jeringa Triple y 3 por actividades del paciente después del procedimiento quirúrgico. Conclusiones: El enfisema subcutáneo puede tener efectos graves y potencialmente mortales si no es tratado adecuadamente. Se debe tener un especial cuidado con el uso de piezas de mano de alta velocidad. Se deben dar indicaciones claras a los pacientes después de los procedimientos quirúrgicos.

PALABRAS CLAVE: Enfisema; enfisema subcutaneo; enfisema mediastínico.

\section{SUMMARY}

Objective: Determine the most frequent cause of subcutaneous emphysema in oral surgery, as well as risk factors, diagnosis and treatment. Material and methods: Comprehensive literature search through PubMed \& Cochrane from 2009 to 2018. Surgical procedures reports were used. Cases caused by trauma and root canals were excluded. We found 21 reports that met our inclusion criteria. Results: It was found that 14 of the 21 cases were related to the use of high-speed handpiece. Other cases included: 4 for the use of the Air Syringe and 3 for patient activities after the surgical procedure. Conclusions: Subcutaneous emphysema can have serious and life-threatening effects if not treated properly. Special care must be taken with the use of high-speed handpieces. Clear instructions should be given to patients after surgical procedures.

KEY WORDS: Emphysema; subcutaneous emphysema; mediastinal emphysema. 


\section{INTRODUCCIÓN}

El enfisema subcutáneo es una complicación poco frecuente y si llega a presentarse puede llegar a ser grave. Se puede presentar durante o después de realizar los procedimientos odontológicos, entre ellos la cirugía oral. De los procedimientos de cirugía oral, el más frecuente es la extracción de los terceros molares inferiores impactados o incluidos. Durante este procedimiento se realizan colgajos a grosor total, separando el periostio del hueso. El enfisema es una afección que se caracteriza por la presencia de aire debajo de los tejidos subcutaneos, lo que provoca inflamación, crepitación a la palpación y la posibilidad de propagación del aire a través de los planos faciales hacia los espacios profundos como el periorbitario, mediastínico, pericárdico y/o torácico $(1,2)$.

Durante el procedimiento de la extracción de las terceras molares inferiores, se utiliza las piezas mano de alta velocidad para realizar la osteotomía y odontosección, el uso de estos instrumentos durante el procedimiento quirúrgico puede permitir el paso aire hacia estos espacios faciales provocando un enfisema subcutáneo, esto puede ocasionar la diseminación de microorganismos que pueden provocar infecciones severas que amenazan la vida del paciente.

El enfisema subcutaneo generalmente remite en unos días, aunque es importante señalar que para contrarrestar la diseminación de microorganismos se debe administrar terapia antibiótica y dependiendo de la extensión de su diseminación incluso se tendría que hospitalizar al paciente.

El objetivo del presente estudio fue determinar la causa más frecuente descrita en la literatura del enfisema sub-cutáneo durante los procedimientos de Cirugía oral.

\section{MATERIAL Y METODOS}

Considerando que en el año 2009 se publicó un estudio retrospectivo realizado por McKenzie WS y Rosenberg $M$ donde se revisaron 32 informes de enfisema subcutáneo de 1993 al 2008(1), se realizó una búsqueda en la literatura médica y odontológica registrada en PubMed \& Cochrane utilizando las palabras clave "subcutaneous emphysema", "Emphysema AND dental " $\mathrm{y}$ "emphysema AND oral surgery.

Limitando la búsqueda a publicaciones en odontología de reportes de enfisema subcutáneo desde el 2009 hasta el 2018, como una continuación de la compilación realizada de McKenzie WS y Rosenberg M. Como criterios de inclusión, se consideraron las publicaciones sobre procedimientos quirúrgicos orales (1).

No se consideraron los casos de enfisema subcutáneo ocasionados por trauma y tratamientos de conductos. Se encontró 21 reportes de casos que cumplían con los criterios de inclusión.

\section{RESULTADOS}

Se incluyeron 21publicaciones de reportes de caso en esta revisión (Tabla 1), que cumplían con los criterios de inclusión. La edad de los pacientes oscila entre los 12 y 54 años de edad. De los cuales 8 fueron varones y 13 mujeres.

Se encontró que 14 de estos casos estaban asociados a procedimientos quirúrgicos de exodoncia de terceros molares. De los restantes: 4 por exodoncia de otras piezas dentarias y solo 1 caso no específica la pieza a la cual se le hizo la exodoncia. Asimismo se encontró 1 caso reportado por coronectomia de tercera molar y 1 caso asociado a tratamiento con aire abrasivo para peri-implantitis con dispositivo escaler. (tabla 2).

Se pudo determinar además que 14 de los 21 casos estaban relacionados con el uso de turbina accionada por aire. Otros casos incluyeron: 4 por el uso de aire comprimido de la Jeringa Triple y 3 por actividades del paciente después del procedimiento quirúrgico. (tabla 3)

Asimismo el examen auxiliar de elección para determinar su distribución fue la Tomografía Axial Computarizada (TAC). En la tabla 1 se puede apreciar cuales fueron los espacios comprometidos, a excepción de un caso que solo fue manejado con Ortopantografia.

De los 21 casos, 17 fueron manejados con tratamiento antibiótico. 2 no reportaron el manejo y a 2 casos no le fue administrado antibiótico. No se reportó ninguna complicación. 
Tabla 1. Artículos de revisión y resultados

\begin{tabular}{|c|c|c|c|c|c|c|c|c|c|}
\hline PAIS Y AÑO & EDAD & SEXO & $\begin{array}{c}\text { PIEZA } \\
\text { DENTARIA }\end{array}$ & PROCEDIMIENTO & CAUSAAPARENTE & SINTOMATOLOGIA & DISTRIBUCION (TAC) & $\begin{array}{l}\text { ANTIBIOTICO DE } \\
\text { ELECCION }\end{array}$ & $\begin{array}{l}\text { ARTICULO DE } \\
\text { REFERENCIA }\end{array}$ \\
\hline $\begin{array}{l}\text { ESPAÑA } \\
(2008)\end{array}$ & 23 & $\mathrm{~F}$ & 48 & EXODONCIA & $\begin{array}{l}\text { USO DE TURBINA } \\
\text { ACCIONADA POR } \\
\text { AIRE. }\end{array}$ & $\begin{array}{l}\text { DOLOR CERVICAL Y } \\
\text { CENTROTORAXICO, } \\
\text { ODINOFAGIA. }\end{array}$ & $\begin{array}{l}\text { CERVICAL, } \\
\text { MEDIASTINO, } \\
\text { PERICARDICO. }\end{array}$ & $\begin{array}{l}\text { AMOXICILINA } \\
\text { CON AC. } \\
\text { CLAVULANICO } \\
\text { PROFILACTICO. }\end{array}$ & 3 \\
\hline $\begin{array}{l}\text { ITALIA } \\
(2008)\end{array}$ & 21 & M & 18283848 & EXODONCIA & $\begin{array}{l}\text { USO DE TURBINA } \\
\text { ACCIONADA POR } \\
\text { AIRE. }\end{array}$ & $\begin{array}{l}\text { AGITACION. AUMENTO } \\
\text { DE VOLUMEN EN } \\
\text { ZONA BAJA DE CARA Y } \\
\text { CUELLO. }\end{array}$ & $\begin{array}{l}\text { NEUMOTORAX, } \\
\text { PERICARDICO, } \\
\text { MEDIASTINO. }\end{array}$ & NO REPORTO & 4 \\
\hline $\begin{array}{l}\text { TAIWAN } \\
(2009)\end{array}$ & 32 & $\mathrm{~F}$ & 38 & EXODONCIA & $\begin{array}{l}\text { USO DE TURBINA } \\
\text { ACCIONADA POR } \\
\text { AIRE. }\end{array}$ & $\begin{array}{l}\text { DOLOR EN CARA } \\
\text { Y CUELLO LADO } \\
\text { IZQUIERDO. }\end{array}$ & $\begin{array}{c}\text { INFRATEMPORAL } \\
\text { IZQUIERDO, ESPACIO } \\
\text { PTERIGOMANDIBULAR, } \\
\text { GENIANO, } \\
\text { MASETERINO, PARA Y } \\
\text { RETRO FARINGEO. }\end{array}$ & $\begin{array}{c}\text { AMPICILINA } \\
\text { SULBACTAM } \\
\text { 1.5GRS C/6HRS. }\end{array}$ & 5 \\
\hline JAPON (2009) & 40 & $\mathrm{~F}$ & 48 & EXODONCIA & $\begin{array}{l}\text { USO DE TURBINA } \\
\text { ACCIONADA POR } \\
\text { AIRE. }\end{array}$ & $\begin{array}{l}\text { AUMENTO DE } \\
\text { VOLUMEN DE REGION } \\
\text { GENIANA, HACIA LA } \\
\text { REGION CERVICAL Y } \\
\text { SUBCUTANEA. }\end{array}$ & $\begin{array}{c}\text { ESPACIO } \\
\text { SUBMANDIBULAR } \\
\text { DERECHO, } \\
\text { PARAFARINGEO, } \\
\text { CAROTIDO, } \\
\text { RETROFARINGEO, } \\
\text { CERVICAL, } \\
\text { PRETRAQUEAL, } \\
\text { MEDIASTINO SUPERIOR. }\end{array}$ & $\begin{array}{l}\text { AMPICILINA 2GRS } \\
\text { AL DIA POR } 4 \\
\text { DIAS. }\end{array}$ & 6 \\
\hline $\begin{array}{l}\text { GRECIA } \\
(2010)\end{array}$ & 29 & M & 48 & EXODONCIA & $\begin{array}{c}\text { AIRE } \\
\text { COMPRIMIDO DE } \\
\text { JERINGA TRIPLE. }\end{array}$ & $\begin{array}{l}\text { DISNEA Y DOLOR EN } \\
\text { EL PECHO. }\end{array}$ & $\begin{array}{l}\text { REGIONES PROFUNDAS } \\
\text { DESDE LA MANDIBULA } \\
\text { HACIA EL MEDIASTINO. }\end{array}$ & NO REPORTO & 7 \\
\hline USA (2011) & 21 & M & 18283848 & EXODONCIA & $\begin{array}{l}\text { PENETRACION DE } \\
\text { AIRE A TRAVEZ DE } \\
\text { Hx.Qx POR INFLAR } \\
\text { GLOBO. }\end{array}$ & $\begin{array}{c}\text { AUMENTO DE } \\
\text { VOLUMEN FACIAL, } \\
\text { DIFICULTAD PARA } \\
\text { DEGLUTIR. }\end{array}$ & PERIORBITAL. & $\begin{array}{l}\text { SI, NO REFIERE } \\
\text { ATB. }\end{array}$ & 8 \\
\hline IRAN (2011) & 16 & M & 37 & EXODONCIA & $\begin{array}{l}\text { USO DE TURBINA } \\
\text { ACCIONADA POR } \\
\text { AIRE. }\end{array}$ & $\begin{array}{l}\text { CREPITACION } \\
\text { Y AUMENTO } \\
\text { DE VOLUMEN } \\
\text { PERIORBITAL, } \\
\text { CERVICAL Y } \\
\text { TORAXICO. }\end{array}$ & $\begin{array}{c}\text { PERIORBITAL, } \\
\text { CERVICAL, TORAXICA, } \\
\text { MEDIASTINO. }\end{array}$ & $\begin{array}{l}\text { CLINDAMICINA + } \\
\text { CEFTAZIDIMA. }\end{array}$ & 9 \\
\hline $\begin{array}{l}\text { ITALIA } \\
\text { (2011) }\end{array}$ & 25 & $\mathrm{~F}$ & 48 & EXODONCIA & $\begin{array}{l}\text { USO DE TURBINA } \\
\text { ACCIONADA POR } \\
\text { AIRE. }\end{array}$ & $\begin{array}{l}\text { EDEMA EN EL } \\
\text { BORDE INFERIOR } \\
\text { DE LA MANDIBULA, } \\
\text { INCAPACIDAD } \\
\text { DE ABRIR EL OJO } \\
\text { DERECHO. }\end{array}$ & $\begin{array}{l}\text { LATERO CERVICAL, } \\
\text { CARRILLOS Y REGION } \\
\text { ORBITAL. }\end{array}$ & $\begin{array}{l}\text { AMPICILINA } \\
\text { SULBACTAM 3GR } \\
\text { AL DIA POR } 5 \\
\text { DIAS. }\end{array}$ & 10 \\
\hline $\begin{array}{l}\text { TAIWAN } \\
\text { (2012) }\end{array}$ & 25 & $\mathrm{~F}$ & 28 & EXODONCIA & $\begin{array}{c}\text { AIRE } \\
\text { COMPRIMIDO DE } \\
\text { JERINGA TRIPLE. }\end{array}$ & $\begin{array}{c}\text { EDEMA } \\
\text { SUBMANDIBULAR, } \\
\text { CUELLOY } \\
\text { SUPRACLAVICULAR. }\end{array}$ & $\begin{array}{l}\text { REGION MANDIBULAR, } \\
\text { MEDIASTINO, } \\
\text { PERICARDIOY } \\
\text { NEUMOTORAX. }\end{array}$ & $\begin{array}{c}\text { CEFALOSPORINA } \\
\text { 1ERA } \\
\text { GENERACION EV } \\
\text { + CEFALOSPORINA } \\
\text { 1ERA } \\
\text { GENERACION VO } \\
\text { POR } 5 \text { DIAS. }\end{array}$ & 11 \\
\hline USA (2013) & 41 & $\mathrm{~F}$ & 47 & EXODONCIA & $\begin{array}{l}\text { USO DE TURBINA } \\
\text { ACCIONADA POR } \\
\text { AIRE. }\end{array}$ & $\begin{array}{c}\text { AUMENTO DE } \\
\text { VOLUMEN } \\
\text { HEMIFACIAL, } \\
\text { CREPITACION EN } \\
\text { CABEZA, CUELLO Y } \\
\text { PARTE SUPERIOR DEL } \\
\text { PECHO. }\end{array}$ & $\begin{array}{l}\text { CERVICO FACIAL, } \\
\text { MEDIASTINO. }\end{array}$ & $\begin{array}{l}\text { SI, NO REFIERE } \\
\text { ATB. }\end{array}$ & 12 \\
\hline $\begin{array}{l}\text { ESPAÑA } \\
(2014)\end{array}$ & 12 & M & - & EXODONCIA & $\begin{array}{l}\text { MANIOBRA DE } \\
\text { BOSTEZO }\end{array}$ & $\begin{array}{l}\text { ODINOFAGIA, DOLOR } \\
\text { TORAXICO. }\end{array}$ & $\begin{array}{c}\text { CERVICAL, } \\
\text { SUPRACLAVICULAR, } \\
\text { AXILAR IZQUIERDA, } \\
\text { NEUMOMEDIASTINO. }\end{array}$ & NO INDICO & 13 \\
\hline USA (2014) & 15 & $\mathrm{~F}$ & 28 & EXODONCIA & $\begin{array}{c}\text { USO DE TURBINA } \\
\text { ACCIONADA POR } \\
\text { AIRE. }\end{array}$ & EDEMA PERIORBITAL. & $\begin{array}{c}\text { ORBITAL Y } \\
\text { PERIORBITAL. }\end{array}$ & $\begin{array}{l}\text { SI, NO REFIERE } \\
\text { ATB. }\end{array}$ & 14 \\
\hline $\begin{array}{l}\text { FRANCIA } \\
\text { (2015) }\end{array}$ & 27 & M & 48 & EXODONCIA & $\begin{array}{l}\text { USO DE TURBINA } \\
\text { ACCIONADA POR } \\
\text { AIRE. }\end{array}$ & $\begin{array}{l}\text { DOLOR CUELLO, } \\
\text { SENSACION DE } \\
\text { BURBUJA AIRE. }\end{array}$ & $\begin{array}{c}\text { ANGULO } \\
\text { MANDIBULA, PARA } \\
\text { Y RETRO FARINGEO, } \\
\text { SUPRACLAVICULAR, } \\
\text { AXILAR, BAJO PIEL } \\
\text { A NIVEL DE 1ERA } \\
\text { VERTEBRA LUMBAR, } \\
\text { MEDIASTINO. }\end{array}$ & PRISTINAMYCIN. & 15 \\
\hline $\begin{array}{l}\text { TURQUIA } \\
\text { (2015) }\end{array}$ & 33 & $\mathrm{~F}$ & 48 & EXODONCIA & $\begin{array}{c}\text { USO DE TURBINA } \\
\text { ACCIONADA POR } \\
\text { AIRE. }\end{array}$ & $\begin{array}{c}\text { AUMENTO DE } \\
\text { VOLUMEN BILATERAL } \\
\text { EN CARA Y CUELLO. }\end{array}$ & $\begin{array}{l}\text { ESPACIO CERVICAL, } \\
\text { MEDIASTINO. }\end{array}$ & $\begin{array}{l}\text { AMPICILINA } \\
\text { SULBACTAM EV. }\end{array}$ & 16 \\
\hline $\begin{array}{l}\text { REINO } \\
\text { UNIDO } \\
(2015)\end{array}$ & 48 & $\mathrm{~F}$ & 48 & CORONECTOMIA & $\begin{array}{c}\text { USO DE TURBINA } \\
\text { ACCIONADA POR } \\
\text { AIRE. }\end{array}$ & $\begin{array}{c}\text { AUMENTO DE } \\
\text { VOLUMEN CERVICO } \\
\text { FACIAL BILATERAL. }\end{array}$ & $\begin{array}{c}\text { PERIORBITAL, } \\
\text { NEUMOMEDIASTINO. }\end{array}$ & NO INDICO & 17 \\
\hline
\end{tabular}




TURQUIA
(2016)

Tabla 2. Procedimientos que originaron el enfisema.

\begin{tabular}{lc}
\hline Procedimiento & n \\
\hline Exodoncia de 3eros molares & 14 \\
Exodoncia de otras piezas dentarias & 4 \\
No especifica & 1 \\
Coronectomia & 1 \\
Tratamiento para priimplantitis con aire abrasivo & 1 \\
\hline
\end{tabular}

Tabla 3. Causas que originaron el enfisema.

\begin{tabular}{lc}
\hline Causa aparente & n \\
\hline Turbina accionada por aire (pieza de mano) & 14 \\
Aire comprimido de jeringa triple & 4 \\
Actividades propias del paciente posterior a procedimiento & 3 \\
\hline
\end{tabular}

\section{DISCUSION}

La palabra enfisema se deriva de la palabra griega 'whick', que significa "soplar" (2). Esta es una complicación poco frecuente, pero muy grave que puede ocurrir durante o posterior al tratamiento odontológico. Aunque el enfisema subcutáneo rara vez causa morbilidad a largo plazo, el reconocimiento precoz y el tratamiento adecuado son fundamentales para prevenir la progresión y sus complicaciones asociadas $(1,25)$.
En 1900, Turnbull describió el primer caso de un paciente con enfisema subcutáneo y pneumomediastino posterior a la exodoncia de una premolar $(22,23)$. En 1995, Heyman y Babayof publicaron una revisión de la literatura en la cual describieron 74 casos de enfisema subcutáneo posterior a tratamientos odontológicos ocurridos entre los años 1960-1993. Ellos reportaron que el $56 \%$ de los casos estuvo relacionado a piezas de mano de alta velocidad (42 de los 74 casos). Asimismo de los 74 casos reportados 33 fueron producidos por extracciones dentales y 27 de estas fueron de $3^{\circ}$ molares. 
Asimismo, el enfisema en los espacios retrofaringeos y mediastinico por diseminación de aire ocurrió en un $35 \%$ de los casos, a consecuencia de tratamientos de exodoncia $(1,22,26)$. Un estudio retrospectivo publicado en 2009 por McKenzie WS y Rosenberg M revisó 32 reportes de caso de enfisema subcutáneo en la práctica Odontológica desde 1993 a 2008, donde el $50 \%$ (16 de los 32 casos), fueron asociados al uso de piezas de mano de alta velocidad (1).

La causa principal de esta complicación es la presión de aire en exceso originado por la pieza de alta velocidad, sobre todo durante la exodoncia de terceras molares mandibulares $(20,22)$. Esto se debe a que el aire es forzado, bajo presión, a los espacios faciales. Una vez que el aire está debajo del tejido, la disección puede ocurrir a lo largo del tejido conectivo relativamente delicado que une los planos musculares adyacentes. La comunicación de los espacios faciales permite que el aire introducido desde la región mandibular se disemine a los espacios retrofaríngeo, mediastínico, pericárdico o torácico (1).

Sin embargo, en nuestra revisión también se encontró que el uso de aire comprimido de la jeringa triple durante el procedimiento quirúrgico puede causar esta complicación $(7,11,18)$. Heyman y Babayof encontraron que $36 \%$ de los enfisemas subcutáneos, fueron asociados a los equipos de jeringas de aire (27 de los 75 casos) (26). A su vez, McKenzie WS y Rosenberg M reportaron cuatro casos ocasionado por jeringa de aire, de los cuales tres estaban relacionados a tratamiento de conductos. Ninguno asociado a procedimientos quirúrgicos (1).

La literatura reporta que, además, puede ser inducido por el paciente al toser, soplar con fuerza, fumar o vomitar después de un procedimiento quirúrgico (1). En esta revisión encontramos que también se originan por inflar globos después del procedimiento, por maniobras de bostezo, por entrenamiento físico posterior a exodoncia compleja y por el uso de aire abrasivo para tratamiento de periimplantitis $(8,13,20,21)$.

Los signos y síntomas de presentación del enfisema subcutáneo varían después de un procedimiento. Mientras que algunos casos reportan signos casi inmediatamente, otros casos tomaron minutos u horas después de que el procedimiento se completó. Los pacientes con enfisema subcutáneo muestran edema marcado e incomodidad cuando el aire ha penetrado en el tejido subcutáneo. Puede manifestarse alteraciones respiratorias si el enfisema subcutáneo se ha diseminado a los espacios paratraqueal, mediastínico o torácico (1).

Los antibióticos son administrados en casos confirmados de enfisema debido a que el aire introducido desde la cavidad oral tiene la capacidad de llevar microorganismos que potencialmente pueden ocasionar una infección la cual puede conllevar a una celulitis o una fasceitis necrotizante (24). En la revisión de McKenzie y Rosenberg, 20 de 32 pacientes recibieron antibiótico después de diagnosticado el enfisema $(1,24)$. En nuestra revisión, 18 de 21 recibieron tratamiento antibiótico, 2 no lo registraron en su reporte y 2 no recibieron tratamiento antibiótico. En ambos casos donde no se indico tratamiento antibiótico, se determino una terapia expectante debido a la buena condición sistémica del paciente. Es importante señalar que ninguno reporto alguna complicación infecciosa $(4,7,17)$.

Como conclusión, es importante seguir las recomendaciones del fabricante sobre el uso y el mantenimiento adecuados de la turbina accionada por aire para prevenir esta complicación, asimismo evitar el uso de aire comprimido de la jeringa triple para secar el lecho quirúrgico. Es importante realizar un examen imagenologico del paciente (TAC) tan pronto como se detecte el enfisema subcutáneo para determinar la extensión y la ubicación del enfisema; así como indicar las instrucciones postoperatorias después de un procedimiento odontológico o quirúrgico de forma clara y concisa. Deben ser escritos y explicados a cada paciente.

\section{Correspondencia:}

Elías Cabanillas.

Correo electrónico: Elias.cabanillas@upch.pe.

\section{REFERENCIAS BIBLIOGRAFICAS}

1. McKenzie WS, Rosenberg M. Iatrogenic subcutaneous emphysema of dental and surgical origin: a literature review. J Oral Maxillofac Surg. 2009; 67(6):1265-8.

2. Mishra L, Patnaik S, Patro S, Debnath N, Mishra S. Iatrogenic subcutaneous emphysema of endodontic origin - case report with literature review. J Clin Diagn Res. 2014; 8(1):279-81. 
3. García AA, García AL, Domínguez B. Subcutaneous emphysema and pneumomediastinum after dental extraction. Med Clin (Barc). 2008; 130(13):518.

4. Magni G, Imperiale C, Rosa G, Favaro R. Nonfatal cerebral air embolism after dental surgery. Anesth Analg. 2008; 106(1):249-51.

5. Kung JC, Chuang FH, Hsu KJ, Shih YL, Chen CM, Huang IY. Extensive subcutaneous emphysema after extraction of a mandibular third molar: a case report. Kaohsiung J Med Sci. 2009; 25(10):562-6.

6. Arai I, Aoki T, Yamazaki H, Ota Y, Kaneko A. Pneumomediastinum and subcutaneous emphysema after dental extraction detected incidentally by regular medical checkup: a case report. Oral Surg Oral Med Oral Pathol Oral Radiol Endod. 2009; 107(4):e33-8.

7. Pousios D, Panagiotopoulos N, Sioutis N, Piyis A, Gourgiotis S. Iatrogenic pneumomediastinum and facial emphysema after surgical tooth extraction. Ann Thorac Surg. 2010; 89(2):640.

8. Maxwell MG, Thompson KM, Hedges MS. Airway compromise after dental extraction. J Emerg Med. 2011; 41(2):e39-41.

9. Afzali N, Malek A, Attar AH. Cervicofacial emphysema and pneumomediastinum following dental extraction: case report. Iran J Pediatr. 2011; 21(2):253-5.

10. Romeo U, Galanakis A, Lerario F, Daniele GM, Tenore G, Palaia G. Subcutaneous emphysema during third molar surgery: a case report. Braz Dent J. 2011; 22(1):83-6.

11. Chen $\mathrm{CH}$, Chang H, Liu HC, Hung TT, Huang WC. Pneumothorax, pneumomediastinum and pneumopericardium complications arising from a case of wisdom tooth extraction. Rev Port Pneumol. 2012; 18(4):194-7.

12.Elia F, Laface B, Pagnozzi F, et al. Cervicofacial emphysema and pneumomediastinum complicating a dental procedure. J Emerg Med. 2013; 45(5):e179-81.

13. Olaciregui Echenique I, Plazaola Cortázar A, Uriz Monaut JJ, Korta Murua J. Subcutaneous emphysema and pneumomediastinum after dental extraction. An Pediatr (Barc). 2014; 80(3):195-6.

14. Fleischman D, Davis RM, Lee LB. Subcutaneous and periorbital emphysema following dental procedure. Ophthalmic Plast Reconstr Surg. 2014; 30(2):e43-5.
15.Picard M, Pham Dang N, Mondie JM, Barthelemy I. Cervicothoracic subcutaneous emphysema and pneumomediastinum after third molar extraction. J Oral Maxillofac Surg. 2015; 73(12):2286.e1-3.

16. Aslaner MA, Kasap GN, Demir C, Akkaş M, Aksu NM. Occurrence of pneumomediastinum due to dental procedures. Am J Emerg Med. 2015; 33(1):125.1-3.

17. Wong C, Collin J, Hughes C, Thomas S. Surgical emphysema and pneumomediastinum after coronectomy. Br J Oral Maxillofac Surg. 2015; 53(8):763-4.

18. Onal O, Hasdiraz L, Oguskaya F. Iatrogenic Pneumomediastinum and Subcutaneous Emphysema after Mandibular Left First Molar Tooth Extraction. Erciyes Med J. 2016; 38(1): 3840.

19. Tan S, Nikolarakos D. Subcutaneous emphysema secondary to dental extraction: A case report. Aust Dent J. 2017; 62(1):95-97.

20. Gowans K, Patel M, Lewis K. Surgical emphysema: A rare complication of a simple surgical dental extraction without the use of an airdriven rotor. Dent Update. 2017; 44(3):217-20.

21.Lee ST, Subu MG, Kwon TG. Emphysema following air-powder abrasive treatment for periimplantitis. Maxillofac Plast Reconstr Surg. 2018; 40(1):12.

22. Tay YBE, Loh WS. Extensive subcutaneous emphysema, pneumomediastinum, and pneumorrhachis following third molar surgery. Int J Oral Maxillofac Surg. 2018; 47(12):1609-12.

23. Bernal FR, Molina TF, Pascacio CX, Angel HL. Parálisis del XI par craneal secundario a enfisema subcutáneo por uso de pieza de mano de alta velocidad en cirugía de terceros molares. Rev ADM. 2018; 75 (2): 92-97.

24. Mitsunaga S, Iwai T, Kitajima H, et al. Cervicofacial subcutaneous emphysema associated with dental laser treatment. Aust Dent J. 2013; 58(4):424-7.

25. Marmolejo TA, Molano VP. Enfisema oral. Rev ADM. 2011; 68(3):136-39.

26. Heyman SN, Babayof I. Emphysematous complications in dentistry, 1960-1993: an illustrative case and review of the literature. Quintessence Int. 1995; 26(8):535-43. 